\title{
ASPECTOS BIOLÓGICOS E DE \\ 1539 \\ COMPORTAMENTO DOS PRINCIPAIS PARASITOS \\ DE Anthonomus grandis BOH. (COLEOPTERA: CURCULIONIDAE), EM CAMPINAS, SP.
}

\author{
*. Ivo Pierozzi Jr. ${ }^{1}$ e Mohamed E. M. Habib ${ }^{2}$
}

\begin{abstract}
Biological and Behavioral Aspects of Anthonomus grandis Boh. (Coleoptera: Curculionidae) Main Parasitoids, in Campinas, SP.

Biological and behavioral aspects of four main species of boll weevil, Anthonomus grandis Boh. hymenopteran ectoparasitoids, occurring in the Campinas region, state of São Paulo, Brazil, were studied under laboratory conditions. Bracon vulgaris Ashmead (Hymenoptera, Braconidae) showed to be easily adapted to artificial rearing conditions, Females oviposited an average of 2.08 eggs/host; egg incubation period varied from 24 to 36 hours; larvae passed through four instars and completed their development in 4.0 days; the same duration time was observed for pupal stage. The longevity of mated adults was in average 41.11 days for females and 31.72 days for males. Last instar larvae of Plodia interpunctella (Hueb.) (Lepidoptera, Pyralidae) were used with success as alternative host, in the absence of $A$. grandis, during the winter. Informations about three other parasitoids species, Bracon sp., Cataloccus grandis (Burks) (Hymenoptera, Pteromalidae) e Eupelmus cushmani (Hymenoptera, Eupelmidae) are also presented.
\end{abstract}

KEY WORDS: Insecta, boll weevil, Bracon spp., Catolaccus grandis, Eupelmus cushmani, biological control.

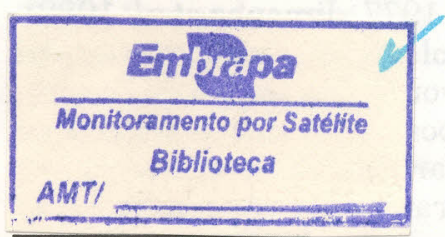

Recebido em 06/05/92.

1NMA_EMBRAPA - Caixa Postal, 491, 13.001-970, Campinas, SP. 2Departamento de Zoologia/IB/UNICAMP, Caixa Postal 6109, 13.081970, Campinas, SP. 


\section{RESUMO}

Aspectos da biologia e do comportamento de quatro espécies de himenópteros ectoparasitos de Anthonomus grandis Boh., o bicudo do algodoeiro, ocorrendo na região de Campinas, SP, foram estudados em condições de laboratório. Bracon vulgaris Ashmead (Hymenoptera, Braconidae) revelou grande adaptabilidade às condições artificiais de criação. As fêmeas deste braconídeo ovipõem em média, 2,08 ovos/ hospedeiro; o período de incubação dos ovos variou de 24 a 36 horas; as larvas passaram por quatro estádios, completando seu desenvolvimento em 4,0 dias, tempo igualmente observado para o estágio pupal. A longevidade de adultos acasalados foi em média, 41,11 dias para fêmeas e 31,72 para machos. Durante a entressafra, na ausência de $A$. grandis, larvas de último estádio de Plodia interpunctella (Hueb.) (Lepidoptera Pyralidae) foram usadas como hospedeiro alternativo, revelando-se adequadas. Informações de outras três espécies de parasitos, Bracon sp., Catoloccus grandis (Burks) (Hymenoptera, Pteromalidae) e Eupelmus cushmani (Hymenoptera, Eupelmidae) também são apresentadas.

PALAVRAS-CHAVE: Insecta, bicudo do algodoeiro, Bracon spp., Catolaccus grandis, Eupelmus cushmani, controle biológico.

\section{INTRODUÇÃO}

Desde a constatação de Anthonomus grandis Boh., o bicudo do algodoeiro, na região de Campinas, SP (Habib \& Fernandes 1983), trabalhos têm demonstrado a ocorrência de parasitos desta praga nas lavouras de algodão (Habib et al. 1984, Pierozzi Jr. et al. 1984, Pierozzi Jr. 1985, Pierozzi Jr. et al. 1985, Pierozzi Jr. \& Queiroz 1985, Pierozzi Jr. \& Habib 1986a, 1986b, Pierozzi Jr. 1989). Dentre eles, os himenópteros ectoparasitos dos estágios imaturos do curculionídeo são os inimigos naturais mais significantes. Os braconideos, notadamente Bracon spp., têm merecido atenção especial devido sua abundância (Pierozzi Jr. 1985, 1989). Esta última razão, aliada ao potencial destes parasitos como agentes de controle biológico (Folsom 1936, Adams et al. 1969, Cross et al. 1969, Bradleigh et al. 1976, Henson et al. 1977, Jimenez et al. 1982), estimularam a realização de investigações biológicas e de comportamento das principais espécies detectados nas lavouras da região de Campinas, SP. Além disto, pretendeu-se revelar as possibilidades de adaptação de Bracon vulgaris Ashmead a condições artificiais de criação para programas de liberação e manutenção do braconideo em condições de campo.

\section{MATERIAL E METODOS}

Para a criação dos parasitos, botões florais e frutos do algodoeiro, infestados por $A$. grandis, foram coletados, semanalmente, de lavouras do município de Campinas (latitude $22^{\circ} 35^{\prime}-23^{\circ} 05^{\prime} \mathrm{S}$ e longitude $46^{\circ} 55^{\prime}$ - $\left.47^{\circ} 35^{\prime} \mathrm{W}\right)$, durante o ciclo do algodão. Estas estruturas levadas ao laboratório, eram examinadas para detecção de larvas do curculionídeo parasitadas. As larvas dos himenópteros eram deixadas sobre seus hospedeiros, ainda dentro de seus sítios de desenvolvimento e mantidos em frascos de vidro $(10 \mathrm{~cm}$ x $15 \mathrm{~cm}$ de altura, a abertura tampada com pano transparente e fundo com papel de filtro) até que completassem seu desenvolvimento e empupassem. As pupas eram separadas em placas de Petri até a emergência dos adultos. Bracon sp., os pteromalídeos e os eupelmideos foram mantidos em frascos de plástico (22 cm x $14 \mathrm{~cm})$. Os adultos de $B$. vulgaris, coletados no campo ou emergidos no laboratório, foram colocados numa caixa de madeira $(60 \mathrm{~cm} \times 42 \mathrm{~cm} \times 32 \mathrm{~cm})$ revestida com pano transparente. Ofereceu-se solução de água e mel a 10\%, em um pedaço de algodão embebido nesta solução e colocado em vidros presos no teto da caixa ou sobre recipientes de folha de alumínio, depositados no fundo dos frascos. Botões florais de algodão contendo larvas de $A$. grandis de último estádio eram oferecidos, diariamente, como substrato para oviposição. A criação e manutenção dos parasitos foi realizada em sala climatizada $\left(25 \pm 2{ }^{\circ} \mathrm{C} ; 70 \pm 10 \%\right.$ U.R.) e com fotoperiodo natural.

Dados de biologia e comportamento de $B$. vulgaris foram obtidos observando-se fêmeas que, pousavam sobre botões florais. O comportamento de oviposição foi observado e registrado até que a fêmea abandonasse o substrato. Os botões foram examinados e aqueles com ovos do parasito, foram separados em grupos de 20 unidades. Seu conteúdo foi examinado e a largura da cápsula cefálica das larvas foi medida com uma ocular micrométrica para determinação do número dos estádios larvais e do índice de crescimento do braconídeo.

Os adultos obtidos dos botões não destruídos foram separados, alimentados e sua longevidade registrada.

No período entre-safras, para a manutenção da criação dos parasitos, foram oferecidas larvas de $P$. interpunctella. Tais larvas foram colocadas vivas em peciolo seco de mamona, previamente sulcados e suas extremidades tampadas com algodão. Esses pecíolos foram inpregnados com extrato de botões florais de algodoeiro para serem atrativos às fêmeas.

\section{RESULTADOS E DISCUSSÃO}

A fêmea do parasito $B$. vulgaris foi atraída imediatamente para os
tões florais de algodão e observou-se que tinham atividade reprodutiva botões florais de algodão e observou-se que tinham atividade reprodutiva a caminhar, tamborilando as antenas na superfície do botão, parava e introduzia o ovipositor. Esta sequência de comportamento também foi 


\section{RESUMO}

Aspectos da biologia e do comportamento de quatro espécies de himenópteros ectoparasitos de Anthonomus grandis Boh., o bicudo do algodoeiro, ocorrendo na região de Campinas, SP, foram estudados em condições de laboratório. Bracon vulgaris Ashmead (Hymenoptera, Braconidae) revelou grande adaptabilidade às condições artificiais de criação. As fêmeas deste braconídeo ovipõem em média, 2,08 ovos/ hospedeiro; o período de incubação dos ovos variou de 24 a 36 horas; as larvas passaram por quatro estádios, completando seu desenvolvimento em 4,0 dias, tempo igualmente observado para o estágio pupal. A longevidade de adultos acasalados foi em média, 41,11 dias para fêmeas e 31,72 para machos. Durante a entressafra, na ausência de $A$. grandis, larvas de último estádio de Plodia interpunctella (Hueb.) (Lepidoptera, Pyralidae) foram usadas como hospedeiro alternativo, revelando-se adequadas. Informações de outras três espécies de parasitos, Bracon sp., Catoloccus grandis (Burks) (Hymenoptera, Pteromalidae) e Eupelmus cushmani (Hymenoptera, Eupelmidae) também são apresentadas.

PALAVRAS-CHAVE: Insecta, bicudo do algodoeiro, Bracon spp., Catolaccus grandis, Eupelmus cushmani, controle biológico.

\section{INTRODUÇÃO}

Desde a constatação de Anthonomus grandis Boh., o bicudo do algodoeiro, na região de Campinas, SP (Habib \& Fernandes 1983), trabalhos têm demonstrado a ocorrência de parasitos desta praga nas lavouras de algodão (Habib et al. 1984, Pierozzi Jr. et al. 1984, Pierozzi Jr. 1985, Pierozzi Jr. et al. 1985, Pierozzi Jr. \& Queiroz 1985, Pierozzi Jr. \& Habib 1986a, 1986b, Pierozzi Jr. 1989). Dentre eles, os himenópteros ectoparasitos dos estágios imaturos do curculionídeo são os inimigos naturais mais significantes. Os braconideos, notadamente Bracon spp., têm merecido atenção especial devido sua abundância (Pierozzi Jr. 1985, 1989). Esta última razão, aliada ao potencial destes parasitos como agentes de controle biológico (Folsom 1936, Adams et al. 1969, Cross et al. 1969, Bradleigh et al. 1976, Henson et al. 1977, Jimenez et al. 1982), estimularam a realização de investigações biológicas e de comportamento das principais espécies detectados nas lavouras da região de Campinas, SP. Além disto, pretendeu-se revelar as possibilidades de adaptação de Bracon vulgaris Ashmead a condições artificiais de criação para programas de liberação e manutenção do braconídeo em condições de campo.

\section{MATERIAL E MÉTODOS}

Para a criação dos parasitos, botões florais e frutos do algodoeiro, infestados por $A$. grandis, foram coletados, semanalmente, de lavouras do municipio de Campinas (latitude $22^{\circ} 35^{\prime}-23^{\circ} 05^{\prime} \mathrm{S}$ e longitude $46^{\circ} 55^{\prime}$ - $47^{\circ} 35^{\prime}$ W), durante o ciclo do algodão. Estas estruturas levadas ao laboratório, eram examinadas para detecção de larvas do curculionídeo parasitadas. As larvas dos himenópteros eram deixadas sobre seus hospedeiros, ainda dentro de seus sitios de desenvolvimento e mantidos em frascos de vidro ( $10 \mathrm{~cm} \times 15 \mathrm{~cm}$ de altura, a abertura tampada com pano transparente e fundo com papel de filtro) até que completassem seu desenvolvimento e empupassem. As pupas eram separadas em placas de Petri até a emergência dos adultos. Bracon sp., os pteromalídeos e os eupelmideos foram mantidos em frascos de plástico $(22 \mathrm{~cm} \times 14 \mathrm{~cm}) .0$ s adultos de $B$. vulgaris, coletados no campo ou emergidos no laboratório, foram colocados numa caixa de madeira ( $60 \mathrm{~cm} \times 42 \mathrm{~cm} \times 32 \mathrm{~cm}$ ) revestida com pano transparente. Ofereceu-se solução de água e mel a 10\%, em um pedaco de algodão embebido nesta solução e colocado em vidros presos no teto da caixa ou sobre recipientes de folha de alumínio, depositados no fundo dos frascos. Botões florais de algodão contendo larvas de $A$. grandis de último estádio eram oferecidos, diariamente, como substrato para oviposição. A criação e manutenção dos parasitos foi realizada em sala climatizada $\left(25 \pm 2{ }^{\circ} \mathrm{C} ; 70 \pm 10 \%\right.$ U.R.) e com fotoperiodo natural.

Dados de biologia e comportamento de $B$. vulgaris foram obtidos observando-se fêmeas que, pousavam sobre botões florais. O comportamento de oviposição foi observado e registrado até que a fêmea abandonasse o substrato. Os botões foram examinados e aqueles com ovos do parasito, foram separados em grupos de 20 unidades. Seu conteúdo foi examinado e a largura da cápsula cefálica das larvas foi medida com uma ocular micrométrica para determinação do número dos estádios larvais e do índice de crescimento do braconídeo.

Os adultos obtidos dos botões não destruídos foram separados, alimentados e sua longevidade registrada.

No período entre-safras, para a manutenção da criação dos parasitos, foram oferecidas larvas de $P$. interpunctella. Tais larvas foram colocadas vivas em pecíolo seco de mamona, previamente sulcados e suas extremidades tampadas com algodão. Esses pecíolos foram inpregnados com extrato de botões florais de algodoeiro para serem atrativos às fêmeas.

\section{RESULTADOS E DISCUSSÃO}

A fêmea do parasito $B$. vulgaris foi atraída imediatamente para os botões florais de algodão e observou-se que tinham atividade reprodutiva durante todo o período de luz do dia. Uma vez pousada a fêmea começava a caminhar, tamborilando as antenas na superfície do botão, parava introduzia o ovipositor. Esta sequência de comportamento também foi 
verificada a campo. Em botões florais sem larvas de $A$. grandis, a fêmea insistia por alguns minutos na procura, introduzindo o ovipositor em locais diferentes do botão, e não encontrando o hospedeiro, abandonavao, pousando em outro. Uma vez detectada a presença do hospedeiro, este era anestesiado e iniciava-se o ato de oviposição. $\mathrm{O}$ tempo gasto nestas ações variou de 5 minutos a um máximo de 7 horas e meia, com uma média de 1,4 \pm 0,1 horas. Adams et al. (1969), estudando B. mellitor Say, detectaram um intervalo de tempo que variou de 8 a 18 minutos desde a picada até a anestesia total da larva de $A$. grandis e presumem que tanto o anestésico como seu modo de ação sejam similares ao de $B$. hebetor (Say).

O ovo de $B$. vulgaris mediu $0,95 \pm 0,02 \mathrm{~mm}$ de comprimento por 0,21 $\pm 0,01 \mathrm{~mm}$ de largura, colocado sobre ou perto da larva hospedeira. Registrou-se um mínimo de 1 e um máximo de 7 ovos/larva de $A$. grandis, e média de $2,08 \pm 0,28$ ovos/hospedeiro. O periodo de incubação dos ovos do braconídeo variou de 24 a 36 horas. As larvas recém-eclodidas localizavam-se por todo o corpo do hospedeiro. O desenvolvimento larva completou-se em cerca de 4 dias, e a larva passa por 4 estádios (Tabela 1). A duração dos três primeiros estádios é de aproximadamente 12 horas para cada, sendo que o quarto estádio dura 48 horas. Após este período, a larva abandona o hospedeiro e dentro do botão tece seu casulo. Em doze horas, a pré-pupa era visivel dentro do casulo já formado e nas 24 horas seguintes, podia se observar aspectos de pupa em transformação, com olhos mais escuros e a última ecdise ocrorrendo nas 12 horas seguintes.

Tabela 1. Largura ( $\mathrm{mm}$ ) da cápsula cefálica dos estádios larvais de Bracon vulgaris e indices de crescimento.

\begin{tabular}{lllll}
\hline \multirow{2}{*}{ Dimensões } & \multicolumn{4}{c}{ Estádios larvais } \\
\cline { 2 - 5 } Média & 0,17 & $2^{0}$ & $3^{\circ}$ & $4^{\circ}$ \\
\pm & \pm & \pm & 0,34 & 0,42 \\
erro padrão & 0,01 & 0,03 & \pm & \pm \\
Máxima & 0,19 & 0,29 & 0,02 & 0,03 \\
Mínima & 0,15 & 0,20 & 0,37 & 0,45 \\
Índice linear & 1,41 & 1,42 & 1,24 & 0,36 \\
Índice médio & & & & \\
de crescimento & & 1,36 & & \\
\hline
\end{tabular}

A pupa dura 3 a 4 dias, nos dois primeiros sua coloração é leitosa passando, posteriormente a coloração castanha como os adultos. $\mathrm{Na}$ pupa, pode-se diferenciar machos e fêmeas pela presença nestas do ovipositor, dobrado e preso dorsalmente, por filamentos cuticulares. Os adultos emergem pela extremidade anterior do casulo e saem dos botões através de um orifício circular $2 \mathrm{~mm}$ de diâmetro. De um total de 120 adultos (59 fêmeas e 61 machos), acasalados e alimentados, a longevidade das fêmeas variou de 9 a 75 dias, com média de 41,11 $\pm 2,89$ dias; para machos 6 a 64 dias, com média de 31,72 $\pm 3,13$ dias. A longevidade maior para fêmeas coincide com as observações de Habib (1968), estudando $B$. hebetor e Patel (1981), investigando Campoletis flavicincta.

O pecíolo seco de mamona contendo as larvas de $P$. interpunctella, atraiu a fêmea do braconideo que manifestou os mesmos comporta, tos anteriormente mencionados. O hospedeiro alternativo, usado para a manutenção de populações do braconídeo, foi adequado pois num período de nove meses, foram obtidas 20 gerações do parasito. A obtenção de razão sexual 1:1 revela a adequação das técnicas de criação pois de acordo com Flanders (1946), De Bach (1981) e Patel (1981), a razão sexual nos parasitos partenogenéticos depende diretamente da quantidade e qualidade do hospedeiro. Após o ato de paralisação, foi observada a fêmea alimentando-se da hemolinfa extravasada do hospedeiro. Tal observação repetiu-se com Bracon sp. observado por Clausen (1972).

Entre os parasitos, Bracon sp. é relativamente maior que o $B$. vulgaris, sendo que as fêmeas de $B$. vulgaris tem um ovipositor com comprimento de $2,46 \pm 0,14 \mathrm{~mm}$, equivalente à metade do corpo; o ovipositor de Bracon sp. mede 6,00 \pm 0,19 mm, equivalendo ao comprimento do seu corpo. O ovo de Bracon sp. difere daquele de $B$. vulgaris por apresentar, na sua extremidade posterior, uma haste recurvada que mede 0,50 $\pm 0,02 \mathrm{~mm}$ de comprimento por 0,11 $\pm 0,01 \mathrm{~mm}$ de largura.

Também foram capturados adultos de Catolaccus grandis (Burks). Estes adultos aceitaram, sob condições de laboratório, larvas e pupas de A. grandis, sobre as quais completaram o desenvolvimento. Este pteromalídeo, monófago (Cross \& Mitchell 1969, Cross \& Chesnut 1971), nunca tinha sido registrado no Brasil e sua distribuição geográfica restringia-se ao sul dos Estados Unidos e América Central. A última espécie de parasito detectada, Eupelmus cushmani, mostrou-se menos freqüente na região. Os três adultos capturados desenvolveram-se em larvas de $A$. grandis em laboratório. Cross \& Chesnut (1971) e Santis (1980) registraram Eutinobothrus brasiliensis (Hambleton), Alabama argillacea (Hueb.) e Pectinophora gossypiella (Saund.), todos insetos pragas do algodoeiro, como outros hospedeiros deste parasito.

\section{LITERATURA CITADA}

Adams, C.H., W.H. Cross \& H.C. Mitchell. 1969. Biology of Bracon mellitor, a parasite of the boll weevil. J. Econ. Entomol. 62: 889-896.

Bradleigh, V.S., R.D. Henson \& C.S. Barfield. 1976. Ovipositional behavior of Bracon mellitor Say (Hymenoptera, Braconidae) a parasitoid of boll weevil (Anthonomus grandis Boh.): I. Isolation and identification of a syntetic releaser of ovipositor probing. J. Chem. Ecol. 2: 431-440. 
Clausen, C.P. 1972. Entomophagous insects. New York, Hafner Publishing Company, 688 p.

Cross, W.H. \& H.C. Mitchell. 1969. Distribution and importance of Heterolaccus grandis as a parasite of the boll weevil. Ann. Entomol. Soc. Am. 62: 235-236.

Cross, W.H. \& T.L. Chesnut. 1971. Arthropod parasites of boll weevil, Anthonomus grandis: 1. An annotated list. Ann. Entomol. Soc. Am. 64: 516-527.

Cross, W.H., W.L. McGovern \& H.C. Mitchell. 1969. Biology of Bracon kirkipatricki and field releases of the parasite for control of the boll weevil. J. Econ. Entomol. 62: 448-454.

De Bach, P. 1981. Control biologico de las plagas de insectos y malas hierbas. México, Comp. Ed. Continental S.A., 949 p.

Flanders, S.E. 1946. Control of sex and sex-limited polymorphism in the Hymenoptera. Quant. Rev. Biol. 21: 135-143.

Folsom, J.W. 1936. Observations on Microbracon mellitor (Say) in relation to the boll weevil. J. Econ. Entomol. 29: 111-116.

Habib, M.E.M. 1968. Histopathological scudies on the effect of Bacillus thuringiensis Berliner on the mediterranean flour moth Anagasta (Ephestia) kuhniella Zeller. Tese de mestrado, Universidade de Alexandria, Egito, 169 p.

Habib, M.E.M. \& W.D. Fernandes. 1983. Anthonomus grandis Boheman (Curculionidae) já está na lavoura algodoeira do Brasil. Rev. Agri. 58: 74.

Habib, M.E.M., W.D. Fernandes, A. Favaro Jr, \& C.F.S. Andrade. 1984. Eficiência do feromônio de agregação e inseticidas quimicos no combate ao bicudo, Anthonomus grandis Boheman, 1843(Coleoptera, Curculionidae). Rev. Agric. 59: 239-251.

Henson, R.D., S.B. Vinson \& C.S. Barfield. 1977. Ovipositional behavior of Bracon mellitor Say, a parasitoid of boll weevi (Anthonomus grandis Boheman): III. Isolation and identification of natural releasers of ovipositor probing. J. Chem. Ecol. 3: 151-158.

Jimenez, J.A., E. Bustamante \& G. Carrero. 1982. Cria y adaptacion del hymenoptero Bracon kirkipatricki, parasito del picudo del algodonero, en condiciones de Colombia. Bogotá, Instituto Colombiano de Agropecuaria, $12 \mathrm{p}$.
Patel, P.N. 1981. Estudos de fatores bióticos de controle natural em populações de Spodoptera frugiperda (J.E. Smith, 1797) (Lepidoptera, Noctuidae). Tese de mestrado, UNICAMP, Campinas, 98 p.

Pierozzi Jr., I. 1985. Ecologia aplicada de Anthonomus grandis grandis Boh., 1843 (Coleoptera, Curculionidae), na região de Campinas, SP Tese de mestrado, UNICAMP, Campinas, $155 \mathrm{p}$.

Pierozzi Jr., I. 1989. Análise e aplicabilidade do complexo ecológico de Anthonomus grandis Boheman, 1843 (Coleoptera, Curculionidae), na região de Campinas, SP. Tese de doutorado. UNICAMP, Campinas, $191 \mathrm{p}$.

Pierozzi Jr., I., M.E.M. Habib \& C.F.S. Andrade. 1984. Ocorrência natural de parasitismo e predação em populações do bicudo Anthonomus grandis Boheman, 1843, p. 163. In Resumos Congresso Brasileiro de Entomologia, 9, Londrina, 346 p.

Pierozzi Jr., I. \& M.C.V. Queiroz. 1985. Comportamento de reprodução em fêmeas de Bracon sp. (Hymenoptera, Braconidae), p. 134. In Resumos Congresso Brasileiro de Zoologia, 12, Campinas, 368 p.

Pierozzi Jr., I., M.E.M. Habib \& C.F.S. Andrade. 1985. Primeiro registro de ocorrência no Brasil de Catolaccus grandis (Burks) (Hymenoptera, Pteromalidae), parasito de Anthonomus grandis Boheman (Coleoptera, Curculionidae), p. 135. In Resumos Congresso Brasileiro de Zoologia, 12, Campinas, $368 \mathrm{p}$.

Pierozzi Jr., I. \& M.E.M. Habib. 1986a. Estudos preliminares de criação em grande escala de Bracon sp. (Hymenoptera, Braconidae), parasito de Anthonomus grandis Boh. (Coleoptera, Curculionidae), p.223. In Resumos Congresso Brasileiro de Entomologia, 10, Rio de Janeiro, $452 \mathrm{p}$

Pierozzi Jr., I. \& M.E.M. Habib. 1986b. Primeiro registro de parasitismo em adultos de Anthonomus grandis Boh. (Coleoptera, Curculionidae) por Hyalomyodes brasiliensis Tow. (Diptera, Tachinidae), p. 241. In Resumos Congresso Brasileiro de Entomologia, 10, Rio de Janeiro, 452.

Santis, L. 1980. Catalogo de los himenopteros brasileños de la série Parasitica incluyendo Bethyloidea. Curitiba, Editora da Universidade Federal do Paraná, 395 p. 